\title{
Cross-Cultural Confirmation of the Type D Personality Construct
}

\author{
Dr. Les Rodriguez, PhD* \\ Johnson \& Johnson Jacksonville, USA
}

*Corresponding author: Dr. Les Rodriguez, $\mathrm{PhD}$, is currently an employee at Johnson \& Johnson Vision, Jacksonville, FL, US, Tel: 904-378-6091; E-mail: Irodr140@its.jnj.com,

Northcentral University, USA.
Received Date: May 09, 2019

Published Date: May 22, 2019

\section{Opinion}

The Type D personality construct is characterized by negative affectivity and social inhibition and is a predictor of poor outcomes in cardiovascular health [1]. Patients with coronary artery disease and Type D personality have a higher rate of mortality and nonfatal myocardial infarction [2]. They also have a four to six times higher risk for anxiety and depression [3], five times higher risk for poor mental health [4] and four times higher risk of recurrent cardiac episodes [5] compared to individuals who are not Type D personality. They are at a significantly higher risk of myocardial infarction, a poorer prognosis following a myocardial infarction [6], and more cardiovascular-related health problems compared to individuals who are not Type D personality [7].

Type D personality is a known risk factor for premature, cardiac-related death [8]. The Type D personality construct has been linked to a number of cardiovascular conditions that strain, weaken and debilitate and ultimately damage the heart muscle as well as serious cardiac outcomes; heart arrhythmias [9], chronic heart failure [10], coronary artery disease [11], hypertension [12], myocardial infarction [4], and peripheral arterial disease [13].

The cross-cultural confirmation of the Type D personality construct is well documented in research literature. The Type D personality construct has been researched and generalized in a number of different cross cultural contexts and populations and its relationship with a number of health conditions examined; Dutch military personnel deployed to Afghanistan [14], Dutch military personnel awaiting deployment [15], domestic violence victims [16], patients with chronic heart failure and peripheral artery disease [17], healthy patients [5], Chinese patients with heart disease [18], Danish patients with heart disease [3], Scottish patients with heart disease [4], work-related problems [6], development of posttraumatic stress disorder [14], irritable bowel syndrome [19], development of stress cardiomyopathy [20], and poor medication adherence [21] among many others.
Some researchers argue that the Type D personality construct does not generalize across all cultures because studies have had limited coverage in countries in Africa, Asia and South America, therefore, generalization of the Type D personality construct to these countries cannot be made [10]. In contrast, Kupper, Pedersen, Hofer, Saner, Oldridge \& Denollet (2013), concluded that their study's ability to establish cross-cultural equivalence in a large sample size, a strength of their study through the HeartQoL project, was a promising outcome because it included many different cultures, countries and languages [10].

The study from the International HeartQoL project conducted a cross-cultural analysis of 6,222 patients with ischemic heart disease from 22 countries to examine the prevalence and validity of the Type D personality construct and demonstrated cross-cultural equivalence [10]. The study included countries from Western Europe (Netherlands, Belgium, Austria, Germany, Switzerland, and France), Southern Europe (Italy, Spain, and Portugal), Northern Europe (Denmark, Sweden, and Norway), Eastern Europe (Ukraine, Poland, Hungary, and Russia), as well as English speaking countries (Australia, Canada, Ireland, United Kingdom, United States [10]. The Type D personality construct has been the subject of research at a global level with studies conducted in many countries such as: Belgium, Iceland, Norway, Ukarine, Korea [22], Canada [23], China [24], Denmark [25], England [26], Germany [27], India [28], Israel [29], Hungary [30], Italy [31], Netherlands [12], Poland [32], Sweden [33], Turkey [34], and Greece [35].

The results of the study from the International HearQoL project of the Type D personality construct showed consistency between countries in Western Europe, Southern Europe, Eastern Europe, Northern Europe, and the United States thus demonstrating that the cross-cultural validity and reliability of the Type D personality construct. 


\section{Author contributions}

The author confirms being the sole contributor of this work and approved it for publication.

\section{Acknowledgement}

None.

\section{Conflict of Interest Statement}

The author declares that the research was conducted in the absence of any commercial or financial relationships that could be construed as a potential conflict of interest.

\section{References}

1. Denollet J, Sys SU, Stroobant N, Rombouts H, Gillebert TC, et al. (1996) Personality as independent predictor of long term mortality in patients with coronary heart disease. The Lancet 347: 417-421.

2. Grande G, Romppel M, Barth J (2012) Association between type D personality and prognosis in patients with cardiovascular disease: A systematic review and meta-analysis. Annals of Behavioral Medicine 43: 299-310.

3. Spindler H, Kruse C, Zwisler AD, Pedersen SS (2009) Increased anxiety and depression in Danish cardiac patients with a type D personality: Cross-validation of the type D scale (DS14). International Journal of Behavioral Medicine 16: 98-107.

4. Williams L, O Connor RC, Grubb NR, O Carroll RE (2011) Type D personalit and illness perceptions in myocardial infarction patients. Journal of Psychosomatic Research 70: 141-144.

5. Nyklíček I, Vorselaars A, Denollet J (2011) Type D personality and cardiovascular function in daily life of people without documented cardiovascular disease. International Journal of Psychophysiology 80 139-142.

6. Mols F, Denollet J (2010) Type D personality in the general population: A systematic review of health status, mechanisms of disease, and workrelated problems. Health and Quality of Life Outcomes 8(9): 1-10.

7. Dannemann S, Matschke K, Einsle F, Smucker MR, Zimmermann K, et al. (2010) Is type D a stable construct? An examination of type D personality in patients before and after cardiac surgery. Journal of Psychosomatic Research 69: 101-109.

8. Denollet J, Schiffer AA, Spek V (2010) A general propensity to psychological Distress affects cardiovascular outcomes: Evidence from research on the type D (distressed) personality profile. Circulation Cardiovascular Quality and Outcomes, Journal of the American Heart Association 3: 546-557.

9. Denollet J, Tekle FB, Vander Voort PH, Alings M, Vanden Broek KC, et al. (2013) Age-related differences in the effect of psychological distress on mortality: type $\mathrm{D}$ personality in younger versus older patients with cardiac arrhythmias. BioMed Research International Pp. 1-7.

10. Widdershoven J, Kessing D, Schiffer A, Denollet J, Kupper N, et al. (2013) How are depression and type D personality associated with outcomes in chronic heart failure patients? Current Heart Failure Reports 10: 244253.

11. Vukovic O, Tosevski DL, Jasovic-Gasic M, Damjanovic A, Zebic M, et al. (2014) Type D personality in patients with coronary artery disease. Psychiatria Danubina 26(1): 46-51.

12. Denollet J (2005) DS14: Standard assessment of negative affectivity social inhibition and Type D personality. Psychosomatic Medicine 67(1): 89-97.

13. Aquarius AE, Smolderen KG, Hamming JF, De Vries J, Vriens PW, et al. (2009) Type D personality and mortality in peripheral arterial disease. Journal of American Medical Association Archives of Surgery 144(8): 728-733.

14. Rademaker AR, Can Zuiden M, Vermetten E, Geuze E (2011) Type D personality and the development of PTSD symptoms: A prospective study. Journal of Abnormal Psychology 120(2): 299-307.
15. Mommersteeg PM, Denollet J, Kavelaars A, Geuze E, Vermetten E, et al. (2011) Type D personality, termperament, and mental health in military personnel awaiting deployment. International Journal of Behavioral Medicine 18: 131-138.

16. Kunst MJJ, Van Bon Martens MJH (2011) Examining the link between domestic violence victimization and loneliness in a Dutch community simple: A comparison between victims and nonvictims by Type D personality. Journal of Family Violence 26: 403-410.

17. Emons WH, Mols F, Pelle A, Smolderen KG, Denollet J, et al. (2012) Type D assessment in patients with chronic heart failure and peripheral arterial disease: Evaluation of the experimental DS(3) scale using item response theory. Journal of Personality Assessment 94(2): 210-219.

18. Yu DS, Thompson DR, Yu CM, Pedersen SS, Denollet J, et al. (2010) Validating the type D personality construct in Chinese patients with coronary heart disease. Journal of Psychosomatic Research 69: 111-118.

19. Sararoudi RB, Afshar H, Adibi P, Daghaghzadeh H, Fallah J, et al. (2011) Type D personality and Quality of life in patients with irritable bowel syndrome. Journal of Research in Medical Science 16(8): 985-992.

20. Compare A, Bigi R, Orrego PS, Proietti R, Grossi E, et al. (2013) Type D personality is associated with the development of stress cardiomyopathy following emotional triggers. Annals of Behavioral Medicine 45(3): 299307.

21. Wu JR, Moser DK (2014) Type D personality predicts poor medication adherence in patients with heart failure in the USA. International Journal of Behavioral Medicine 21: 833-842.

22. Weng CY, Denollet J, Lin CL, Lin TK, Wang WC, et al. (2013) The validty of the type D construct and its assessment in Taiwan. BioMed Central Psychiatry 13(46): 1-9.

23. Williams L, Curren C, Bruce G (2011) Are alexithymia and type D personalit distinct or overlapping constructs? A confirmatory factor analysis of the Toronto alexithymia and type D scales. Personality and Individual Differences 51: 683-686.

24. Xiao Nan Y, Jian Xin Z (2006) Application of the type D personality scale in Chinese college students. Chinese Mental Health Journal 20(5): 313316.

25. Pedersen SS, Denollet J (2004) Validity of the Type D personality construct in Danish post-MI patients and healthy controls. Journal of Psychosomatic Research 57: 265-272.

26. Whitehead DL, Perkins Porras L, Strike PC, Magid K, Steptoe A, et al. (2007) Cortisol awakening response is elevated in acute coronary syndrome patients with type D personality. Journal of Psychosomatic Research 62: 419-425.

27. Grande G, Jordan J, Kümmel M, Struwe C, Schubmann R, et al. (2004) Evalution of the German type D (DS14) and prevalence of the type D personality pattern in cardiological and psychosomatic patients and healthy subjects. Psychotherapie Psychosomatik Medizinishche Psychologie 54(11): 413-422.

28. Verma KB, Khan MI (2007) Social inhibition, negative affectivity, and depression in cancer patients with type D personality. Social Science International 23(1): 114-122.

29.Zohar AH, Denollet J, Ari LL, CloningerCR (2011) The psychometric Properties properties of the DS14 Hebrew and the prevalence of type D personality in Israeli adults. European Journal of Psychological Assessment 27(4): 274-281.

30. Gyorgy P, Sandor R, Ildiko D, Maria K (2006) Psychometric properties of the Hungarian version of the type D scale in a large community survey. Mentahigiene es Pszichoszomatika 7 (3): 225-230.

31. Gremigni P, Sommaruga M (2005) Type D personality, a relevant construct in cardiology: Preliminary validation of the Italian questionnaire. Psicoterapia Cognitiva e Comportamentale 11(1): 7-18.

32. Oginska Bulik N (2006) Occupational stress and its consequences in healthcare professionals: The role of type D personality. International Journal of Occupational Medicine and Environmental Health 19(2): 113122. 
33. Broström A, Strömberg A, Mårtensson J, Ulander M, Harder L, et al. (2007) Association of type D personality to perceived side effects and adherence in CPAP treated patients with OSAS. Journal of Sleep Research 16(4): 439-447.

34. Aytekin Alçelik, Osman Yıldırım, Fatih Canan, Mustafa Eroğlu, Gülali Aktaş, et al. (2012) A preliminary psychometric evaluation of the type D personality construct in Turkish hemodialysis patients. Journal of Mood Disorders 2(1): 1-5.

35. Christodoulou C, Douzenis A, Mommersteeg PMC, Rallidism L, Poulios A, et al. (2013) A case-control validation of type D personality in Greek patients with stable coronary heart disease. Annals of General Psychiatry 12(38): 1-7. 